\section{Organising an English journal club in the developing world}

\section{J Tucker, X Gao, S Wang, Q Chen, Y Yin, X Chen}

English language journal clubs are fundamental for teaching and collaboration in developing areas

T he medical journal club remains at the heart of evidence based medicine among teaching institutions. Previous reviews have analysed the importance of journal clubs in English speaking nations. ${ }^{1-3}$ A meta-analysis of postgraduate journal clubs using Cochrane-like criteria for selection of papers established that journal clubs broaden a postgraduate student's sense of clinical epidemiology and biostatistics, reading habits, and using medical literature in clinical situations. ${ }^{4}$ Previous analysis in this area has weighed heavily on the side of English speaking journal clubs in nations where English fluency is assumed. This commentary explores the role of the English journal club outside of areas where English is the mother tongue. Three central questions are discussed:

(1) Why do so few nations where English is the second language have English journal clubs?

(2) What are the relative advantages of promoting English journal clubs in these nations?

(3) How can you establish an English journal club in your hospital?

On a worldwide scale, approximately 325 million people use English as their mother tongue, and at least twice as many people speak English as a second or third language.

While English journal clubs are routinely a part of postgraduate medicine culture in developed countries, they are rare in developing world hospitals and medical training centres. Lack of journal access (print or electronic), lack of time or incentives for doctors to participate, and lack of resources make journal clubs difficult to start in the developing world. Oral English proficiency may also be a problem in many areas, since foreign physicians have few opportunities to speak English. Journal clubs currently in progress are more likely to be in the native tongue, and focus more on purely clinical considerations. As a result, few institutions in the developing world currently have English speaking journal clubs.

Although there are barriers to creating English journal clubs in nations where English is the second language, the benefits of establishing such mental exercises are considerable. Beyond the benefits already discussed, journal clubs provide a valuable opportunity to enhance communication skills and English proficiency within a discipline.

\section{Box 1: Starting an English language journal club where English is the second language}

- All articles chosen must be in English and meet the criteria described above.

- The presenter should be encouraged to read several articles regarding a topic before choosing a single article to share with the group.

- The presenter should arrange an overview of important terms with native language/English translation, providing copies to all members of the journal club at least three days beforehand. Presenters should be encouraged to email papers if more convenient, or post on a web page.

- All members of the journal club should read the paper in advance and bring five questions in English.

- During the journal club, the presenter should show a PowerPoint presentation highlighting important aspects of the study and its design.

- Presenters should encourage the involvement of all members of the journal club, and a professor or facilitator should ensure that all participants use their English language skills.

- Suggestions from students and participants should be incorporated to create a flexible programme.

\section{Learning points}

- While English journal clubs are routinely a part of postgraduate medicine culture in developed countries, they are rare in developing world hospitals and medical training centres.

- A regular English journal club nurtures a deeper sense of international ethical principles alongside enhancing communication skills essential for multicentre studies and other cooperation.

- English language journal clubs are fundamental to increasing language proficiency, ethical understanding, and international exchanges in developing areas. 
presented and compared to international standards referenced in the Declaration of Helsinki, ${ }^{5}$ the ethical requirements of international research will be more deeply understood. A regular English journal club nurtures a deeper sense of international ethical principles alongside enhancing communication skills essential for multicentre studies and other cooperation.

A case study from the National STD Center (Chinese Academy of Sciences, Peking Union Medical College) in Nanjing represents an example from the world's largest developing country. Affiliated with the Peking Union Medical College in Beijing, the Institute coordinates research for masters and doctoral students in the fields of dermatology and sexually transmitted infections. Clinical research is central to its mission as a teaching institution. The library and computer facilities present allow access to all major medical journals and to texts in dermatology and sexually transmitted infections. The National STD Center cooperates with several international research organisations, receiving funds from international agencies including the National Institutes of Health and the National
Institute of Mental Health in the United States. Before 2003, the only didactics provided for research students were lectures from professors, both local and international. During 2003, the National STD Center started the first journal club of its kind at the centre, focusing on sexually transmitted infections and the most current literature. Articles were selected from the English literature based on the following criteria: (1) medical merit and topic relevance; (2) work is current; and (3) study design.

English journal clubs need to be well organised in order to be well attended. The list of suggestions shown in box 1 was compiled by the Chinese National STD Center's weekly journal club in order to help those starting new English journal clubs in nations where English is the second language.

English language journal clubs are fundamental to increasing language proficiency, ethical understanding, and international exchanges in developing areas. Especially in national research centres or institutions with international grants or foreign support, establishing a disciplinary English journal club lays the foundation for a sound collaboration.
Research institutions in the developing world without English journal clubs need to consider the advantages of starting such a programme at their hospital or teaching institution.

Postgrad Med J 2004;80:436-437. doi: 10.1136/pgmi.2004.022657

\section{A...................}

\section{Authors' affiliations}

J Tucker, X Gao, S Wang, Q Chen, Y Yin, $X$ Chen, National Center for STD Control, Nanjing, China

Correspondence to: Dr Joseph Tucker, National Center for STD Control, 12 Jiangwangmiao St, Nanjing, China 210042; Joseph_Tucker@med. unc.edu

\section{REFERENCES}

1 Gibbons AJ. Organising a successful journal club. BMJ 2002;325:s137-8.

2 Gonzalez LS. Referees make journal clubs fun. BMJ 2003;326: 106.

3 Alguire PC. A review of journal clubs in postgraduate medical education. J Gen Intern Med 1998; 13:347-53.

4 Ebbert JO, Montori VM, Schultz HJ. The journal club in postgraduate medical education: a systematic review. Med Teach 2001;23:455-61. 5 World Medical Association. Declaration of Helsinki: ethical principles for medical research involving human subjects. Available at: http:// www.wma.net/e/policy/pdf/17c.pdf (cited 11 March 2004). 